\title{
Dissipative Exciton Transfer in Donor-Bridge-Acceptor Systems: Numerical Renormalization Group Calculation of Equilibrium Properties
}

\author{
Sabine Tornow, ${ }^{1}$ Ning-Hua Tong ${ }^{*},{ }^{2}$ and Ralf Bulla ${ }^{1}$ \\ ${ }^{1}$ Theoretische Physik III, Elektronische Korrelationen und Magnetismus, Universität Augsburg, 86135 Augsburg, Germany \\ ${ }^{2}$ Institut für Theorie der Kondensierten Materie, Universität Karlsruhe, 76128 Karlsruhe, Germany
}

(Dated: September 30, 2018)

\begin{abstract}
We present a detailed model study of exciton transfer processes in donor-bridge-acceptor (DBA) systems. Using a model which includes the intermolecular Coulomb interaction and the coupling to a dissipative environment we calculate the phase diagram, the absorption spectrum as well as dynamic equilibrium properties with the numerical renormalization group. This method is nonperturbative and therefore allows to cover the full parameter space, especially the case when the intermolecular Coulomb interaction is of the same order as the coupling to the environment and perturbation theory cannot be applied. For DBA systems up to six sites we found a transition to the localized phase (self-trapping) depending on the coupling to the dissipative environment. We discuss various criteria which favour delocalized exciton transfer.
\end{abstract}

PACS numbers: 71.27.+a Strongly correlated electron systems, 71.35.Aa Frenkel Excitons and Self-Trapped Excitons, 71.35.Cc Intrinsic Properties of Excitons; Optical Absorption Spectra.

\section{INTRODUCTION}

Exciton transfer belongs to the key processes in many chemical and biological systems, organic based nanostructures and semiconductors [1, 2, 3]. The progress in manufacturing molecular electronic devices, biological hybrid systems, and model systems based on quantum dots, nanoscale molecular aggregates and bio-engineered proteins opens the door to understand these fundamental processes [4] and also to find applications in (bio-) molecular electronics, biosensing, and quantum computation [5].

Excitons are electron-hole pairs which do not transfer charge but energy by deexciting a donor molecule followed by the excitation of an acceptor molecule. The radiationless excitation transfer is caused by dipole and exchange interactions and proceeds via a short lived virtual photon 6]. In this work we consider Frenkel excitons [7] where the exciton is a molecular excitation with an electron in the lowest unoccupied molecular orbital (LUMO) and a hole in the highest occupied molecular orbital (HOMO) on the same molecule. Here the Coulomb coupling of the electron-hole pair is much larger than the hopping matrix element of a single hole or electron. Exciton transfer where the Frenkel exciton concept can be applied, occurs in many bio-molecules, e.g. rhodopsin, porphyrins, blue copper protein, carotenoids, and chlorophylls. A well studied molecule is the light harvesting antenna (LH II) from the bacterial photosystem Rhodopseudomonas Acidophila. It is characterized by a symmetric structure and composed of nine identical units forming a ring. Each unit is composed of a chloro-

*present adress: Department of Physics, Renmin University of China, Beijing 100872, China phyll dimer. The light-harvesting complexes store and transfer excitations with high efficiency.

In the photosynthetic process a LH-II ring absorbs a photon. The excitation is transferred to other LH-II rings and sent via the LH-I ring to the reaction center and then converted to chemical energy. The excitation in the LH-II ring B850 can move over the whole ring - it is delocalized over the ring. In other rings, such as B800, the excitations are usually considered to be more localized [4]. Furthermore, mechanisms exist which dissipate excitation energy to safe the organism from damage [8]. The degree of delocalization depends strongly on the coupling to the vibronic environment and may be crucial for the function of the specific protein.

The interpretation of optical spectra [9] requires a theory which incorporates both static and dynamic disorder. If the fluctuations of the protein environment occur on a much larger time scale than those of the excitonic system, the disorder is regarded as static. Such a static disorder can be treated by a thermodynamic average. The dynamic disorder stems from the coupling of the electronic degrees of freedom to the fluctuations of the environment. In the present paper we will study the effect of dissipation while neglecting static disorder.

A full $a b$ initio quantum chemical calculation of molecules which show exciton transfer reactions is impossible; therefore it is reasonable to investigate the system using simple models which, nevertheless, cover the relevant physics of the problem. The most elementary nontrivial model which describes quantum dissipation is the well studied spin-boson model 10, 11]. It can be viewed as an archetype for modelling the system-environment interaction in bio-molecules in which the electronic degrees of freedom couple to a dissipative environment.

A variety of theoretical methods have been developed to calculate absorption spectra and rates (see e.g., 
12, 13, 14, 15, 16, 17, 18, 19]). Some investigations of exciton transfer systems were based on perturbation theory in the exchange coupling between the excitons or in the coupling of the electronic system to the vibrations. If the exciton-vibrational coupling $\alpha$ is weak compared to the dipole-dipole coupling density-matrix theory is used [3]. If the intermolecular Coulomb coupling $J$ is small we are in the limit of nonadiabatic exciton transfer. Here perturbation theory is applicable which leads to the Förster equations [3].

A key challenge for a theoretical study of exciton transfer is to cover the whole range of possible behaviour, from coherent to incoherent transfer or even localization or self-trapping of the excitations. Here we use the nonperturbative numerical renormalization group (NRG) to calculate equilibrium properties of the exciton system in the full parameter space. We give a detailed study of the phase diagram, dynamic equilibrium properties for chains and rings up to six sites, and the frequency dependent linear absorption spectra of excitons in a dimer and trimer molecule as a function of the coupling to the bosonic bath. The behaviour of the system is governed by the competition between the couplings $J$ and $\alpha$ which determine whether the excitations are delocalized or localized.

The models we are considering here describe general electron-boson systems with a limited number of quantum states on a few sites with a coupling to a (quantum) dissipative environment. We restrict ourselves to donor, acceptor, and bridge molecules with only two electronic levels per molecule, neglecting the spin degree of freedom. If the flux of photons is sufficiently low then the exciton migration in systems such as a pigment network can be satisfactorily modelled by a single excitation. We show that in the single-exciton subspace the multisite electron-boson model can be mapped to a multi-site exciton-boson model. The two-site exciton-boson model is identical to the spin-boson model. The exciton system is coupled to all degrees of freedom of the (protein) environment which is modelled by an infinite set of harmonic oscillators. After the discussion of the various models in Sec. II, we introduce the NRG approach used here in Sec. III. Section IV is devoted to the results for the phase diagram, dynamic properties, and the absorption spectrum. We show how the degree of delocalization depends on the different Coulomb interactions, the coupling to the bosonic bath, and the geometric structure.

\section{MODEL}

In general we describe the problem by a small electronic system like a short chain or ring of molecules with the electronic part $H_{\mathrm{el}}$ coupled via the $H_{\text {el-bath }}$ part to the vibronic degrees of freedom incorporated in $H_{\text {bath }}$ :

$$
H=H_{\text {el }}+H_{\text {el-bath }}+H_{\text {bath }} .
$$

The simplest possible model for the electronic part is a two-state system with the two states corresponding to the electron being located at the donor or at the acceptor site. In this case, the electronic part can be modelled via

$$
H_{\mathrm{el}}=\sum_{i=A, D} \epsilon_{i} c_{i}^{\dagger} c_{i}-t\left(c_{A}^{\dagger} c_{D}+c_{D}^{\dagger} c_{A}\right) .
$$

Apparently, models of this kind where the electrons are allowed to hop between donor and acceptor sites (with hopping matrix element $t$ ) are connected to electron transfer problems. In this paper we do not consider such hopping processes and focus on excitation transfer induced by a two-particle interaction term in the Hamiltonian. The electronic part of the Hamiltonian then takes the following general form

$$
H_{\mathrm{el}}=\sum_{i, l, \sigma} H(i, l, \sigma)+\sum_{i, j ; k, l ; \sigma, \sigma^{\prime}} V\left(i, j ; k, l ; \sigma, \sigma^{\prime}\right),
$$

where the first term represents the local part with on-site energies, on-site Coulomb interactions as well as possible spin-orbit couplings. The second part describes all nonlocal terms, mainly the non-local Coulomb interaction (as a possible extension also the single-particle hopping term). In principle, the parameters of the Hamiltonian eq. (3) can be extracted from quantum chemical calculations [20, 21].

In the current work, we explore excitons in a chain or ring using the Hamiltonian

$$
H_{\mathrm{el}}=\sum_{i k} \epsilon_{i k} c_{i k}^{\dagger} c_{i k}+\sum_{i j l k l^{\prime} k^{\prime}} J_{i j} c_{i k}^{\dagger} c_{j l}^{\dagger} c_{j l^{\prime}} c_{i k^{\prime}} .
$$

The operators $c_{i k}^{(\dagger)}$ denote annihilation (creation) operators for the electrons on site $i$ in the level $k, \epsilon_{i k}$ is the onsite energy and $J_{i j}$ the exchange interaction between site $i$ and $j$. The electronic part of each level is represented by a two-level system. We neglect the single-electron hopping between neighbouring sites as well as spin degrees of freedom. Therefore, the excitations (Frenkel excitons) can only be transferred via the Coulomb coupling. Similar models were discussed, e.g., in 3, 16, 18, 22]

The Hamiltonian eq. (4) describes a coherent motion of the excitation through the whole system; this coherence can be destroyed in the presence of a dissipative environment. Here, the coupling to the environment is due to the change of the dipole moment of the molecule during the transition. Simulations 23] showed that the coupling involves essentially all nuclear degrees of freedom of the protein which have to be described quantum mechanically. Even at physiological temperatures there are many degrees of freedom in proteins with frequencies high enough to make a quantum mechanical description 
necessary 23. We represent the vibrations of the environment by a set of harmonic oscillators similar to the spin-boson model.

The last term in eq. (1) describes the free bosonic bath

$$
H_{\mathrm{bath}}=\sum_{n} \omega_{n} b_{n}^{\dagger} b_{n}
$$

with the bosonic annihilation (creation) operators $b_{n}^{(\dagger)}$. The second term in eq. (1) describes the coupling of the electrons to the bosonic bath

$$
H_{\mathrm{el-bath}}=\sum_{i \sigma} g_{i} n_{i \sigma} \sum_{n} \lambda_{n}\left(b_{n}^{\dagger}+b_{n}\right),
$$

where $\lambda_{n}$ is the coupling strength to the $n$th oscillator. We consider here a dipole coupling so that the sum $\sum_{i} g_{i} n_{i}$ is the polarization operator of the electronic system. The values of the parameters $g_{i}$ will be specified below.

In analogy to the spin-boson model [10, 11], the coupling of the electrons to the bath degrees of freedom is completely specified by the bath spectral function

$$
J(\omega)=\pi \sum_{n} \lambda_{n}^{2} \delta\left(\omega-\omega_{n}\right)
$$

Several parametrizations of $J(\omega)$ have been studied in the literature [10]. For a given system, the bath spectral function can also be calculated using molecular dynamics simulations [17]. Here we restrict ourselves to a simple ohmic spectral function and will use more realistic spectral functions in a future study.

\section{Dimer}

For the dimer (that is the two-site electron-boson model as sketched in Fig. 11) the Hamiltonian reduces to

$$
H_{\text {dimer }}=H_{\mathrm{el}}+H_{\mathrm{el}-\mathrm{bath}}+H_{\mathrm{bath}},
$$

with the electronic part

$$
H_{\mathrm{el}}=\sum_{i=D_{0}, D_{1}, A_{0}, A_{1}} \varepsilon_{i} c_{i}^{\dagger} c_{i}+J\left(c_{D_{0}}^{\dagger} c_{A_{1}}^{\dagger} c_{D_{1}} c_{A_{0}}+h . c .\right)
$$

and the coupling term

$$
H_{\mathrm{el}-\mathrm{bath}}=\sum_{i=D_{0}, D_{1}, A_{0}, A_{1}} g_{i} n_{i} \sum_{n} \lambda_{n}\left(b_{n}^{\dagger}+b_{n}\right) .
$$

The indices $D_{0}, A_{0}$ indicate the ground state on the donor/acceptor and $D_{1}, A_{1}$ the first excited states on the donor/acceptor. For the $g_{i}$ we put $g_{D_{1}}=-g_{D_{0}}=$ $-g_{A_{1}}=g_{A_{0}}=\frac{1}{2}$.

The electronic degrees of freedom in this subspace can be represented by the four-dimensional basis

$$
|i\rangle=\{|0,0\rangle,|1,0\rangle,|0,1\rangle,|1,1\rangle\},
$$

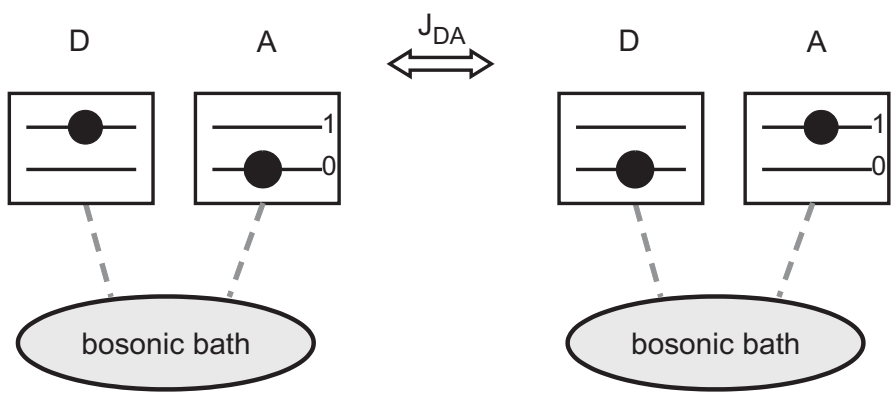

FIG. 1: Schematic view of the two-site electron-boson model. The transfer integral for the exciton transfer between donor (D) and acceptor (A) sites is given by $J$. Dissipation in the exciton transfer process is due to the coupling to a common bosonic bath.

with the notation $|\mathrm{D} ; \mathrm{A}\rangle$ describing the donor/acceptor in the ground state $(D / A=0)$ or in the excited state $(D / A=1)$. Introducing the notation

$$
\hat{Y}=\sum_{n} \omega_{n} b_{n}^{\dagger} b_{n} \quad, \quad \hat{X}=\frac{1}{2} \sum_{n} \lambda_{n}\left(b_{n}^{\dagger}+b_{n}\right),
$$

we arrive at the matrix $M=M_{0}+M_{b}=\left\langle i\left|H_{\text {dimer }}\right| j\right\rangle$ $(i, j=1 . .4)$, where the matrix elements are taken only with respect to the electronic states:

$M_{0}=\left(\begin{array}{cccc}\epsilon_{D_{0}}+\epsilon_{A_{0}} & 0 & 0 & 0 \\ 0 & \epsilon_{D_{1}}+\epsilon_{A_{0}} & J & 0 \\ 0 & J & \epsilon_{D_{0}}+\epsilon_{A_{1}} & 0 \\ 0 & 0 & 0 & \epsilon_{D_{1}}+\epsilon_{A_{1}}\end{array}\right)$,

and

$$
M_{b}=\left(\begin{array}{cccc}
\hat{Y} & 0 & 0 & 0 \\
0 & \hat{Y}-\hat{X} & 0 & 0 \\
0 & 0 & \hat{Y}+\hat{X} & 0 \\
0 & 0 & 0 & \hat{Y}
\end{array}\right)
$$

With $\epsilon_{D_{0}}=\epsilon_{A_{0}}=0$ and $\epsilon_{D_{1}}=\epsilon_{D}, \epsilon_{A_{1}}=\epsilon_{A}$ the eigenvalues of the electronic part $M_{0}$ are

$$
\begin{aligned}
E_{1} & =0 \\
E_{2} & =\epsilon_{D}+\epsilon_{A} \\
E_{3,4} & =\frac{\epsilon_{D}+\epsilon_{A}}{2} \\
& \pm \sqrt{\frac{\left(\epsilon_{D}-\epsilon_{A}\right)^{2}}{4}+J^{2}}
\end{aligned}
$$

The eigenstates with energies $E_{3,4}$ are linear combinations of the basis states $|1,0\rangle$ and $|0,1\rangle$.

The Hamiltonian of the dimer eq. (8) can be decomposed into subspaces of zero, one, and two excitons. For the subspace with one exciton in the dimer, the basis 


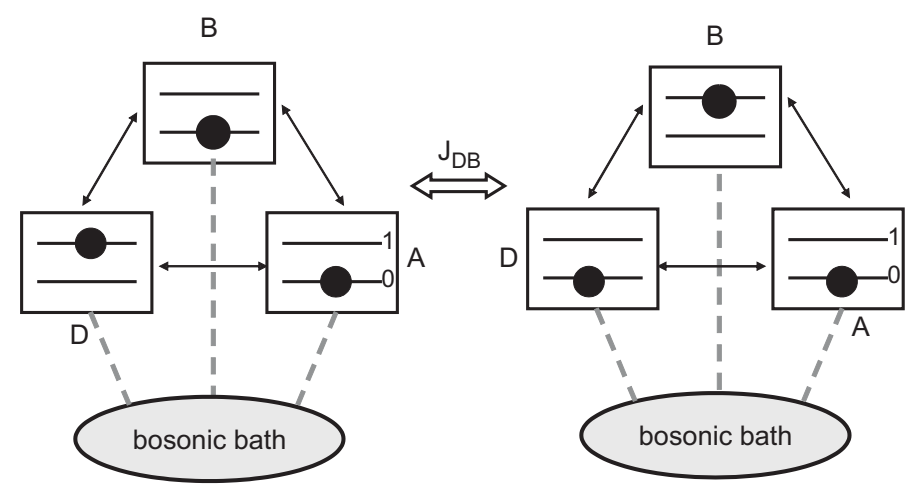

FIG. 2: Schematic view of the three-site electron-boson model. The Coulomb matrix element of excitons between donor (D), bridge (B) and acceptor (A) sites is given by $J$. Dissipation in the exciton transfer process is due to the coupling of the electronic degrees of freedom to a common bosonic bath. The excitation transfer shown in the figure is due to the coupling $J_{D B}$

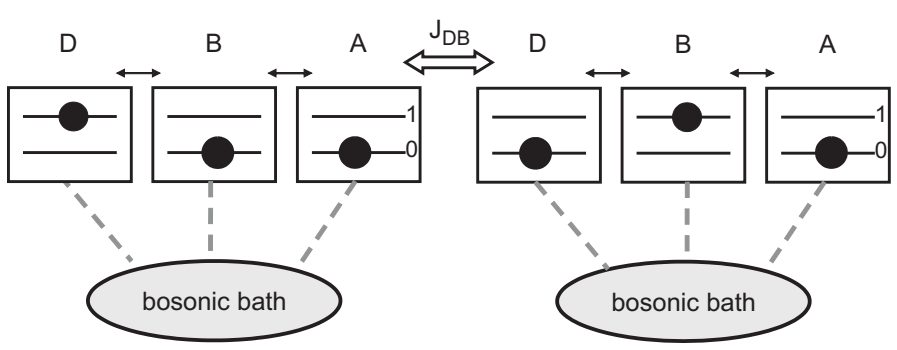

FIG. 3: Schematic view of the three-site (chain) electronboson model.

eq. (11) reduces to $|i\rangle=\{|1,0\rangle,|0,1\rangle\}$ and the Matrix $M$ reads

$$
M=\left(\begin{array}{cc}
\epsilon_{A}+\hat{Y}-\hat{X} & J \\
J & \epsilon_{D}+\hat{Y}+\hat{X}
\end{array}\right)
$$

This matrix allows for an exact mapping onto the spinboson model (for a similar discussion, see Ref. 24). The model is equivalent to eq. (22) with $N=2$.

\section{Trimer}

The Hamiltonian for a donor-bridge-acceptor system in a trimer geometry (see Figs. 2] and [3) takes the form

$$
\begin{aligned}
H_{\mathrm{el}} & =\sum_{i=D_{0}, D_{1}, A_{0}, A_{1}, B_{0}, B_{1}} \varepsilon_{i} c_{i}^{\dagger} c_{i} \\
& +J_{D A}\left(c_{D_{0}}^{\dagger} c_{A_{1}}^{\dagger} c_{D_{1}} c_{A_{0}}+\text { h.c. }\right) \\
& +J_{A B}\left(c_{B_{0}}^{\dagger} c_{A_{1}}^{\dagger} c_{B_{1}} c_{A_{0}}+\text { h.c }\right) \\
& +J_{B D}\left(c_{B_{0}}^{\dagger} c_{D_{1}}^{\dagger} c_{B_{1}} c_{D_{0}}+\text { h.c. }\right) .
\end{aligned}
$$

The electrons are coupled to the bosonic bath via:

$$
H_{\text {el-bath }}=\sum_{i=D_{0}, D_{1}, A_{0}, A_{1}, B_{0}, B_{1}} g_{i} n_{i} \sum_{n} \frac{\lambda_{n}}{2}\left(b_{n}^{\dagger}+b_{n}\right)
$$

For the $g_{i}$ we choose $g_{D_{1}}=-g_{D_{1}}=-g_{A_{0}}=g_{A_{1}}=1$ and $g_{B_{0}}=g_{B_{1}}=0$.

The basis for the electronic degrees of freedom is now composed of eight states:

$|1\rangle=|0,0,0\rangle,|2\rangle=|1,1,1\rangle,|3\rangle=|1,0,0\rangle,|4\rangle=|0,1,0\rangle$, $|5\rangle=|0,0,1\rangle,|6\rangle=|1,1,0\rangle,|7\rangle=|0,1,1\rangle,|8\rangle=|1,0,1\rangle$,

where the 0(1) indicates an occupied first (second) level, respectively, with the notation $|D, B, A\rangle$ for the occupation of donor, bridge, and acceptor molecule. A direct hopping of the exciton is possible from the donor to the acceptor or to the bridge and from the acceptor to the bridge and back (see Fig. 2). The matrix-elements now read:

$$
\begin{aligned}
& \langle 1|H| 1\rangle=\epsilon_{D_{0}}+\epsilon_{B_{0}}+\epsilon_{A_{0}}+\hat{Y}+\left(g_{B_{0}}+g_{D_{0}}+g_{A_{0}}\right) \hat{X}, \\
& \langle 2|H| 2\rangle=\epsilon_{D_{1}}+\epsilon_{A_{1}}+\epsilon_{B_{1}}+\hat{Y}+\left(g_{A 1}+g_{D_{1}}+g_{B 1}\right) \hat{X}, \\
& \langle 3|H| 3\rangle=\epsilon_{A_{0}}+\epsilon_{B_{0}}+\epsilon_{D_{1}}+\hat{Y}+\left(g_{A_{0}}+g_{B_{0}}+g_{D_{1}}\right) \hat{X}, \\
& \langle 4|H| 4\rangle=\epsilon_{D_{0}}+\epsilon_{B_{1}}+\epsilon_{A_{0}}+\hat{Y}+\left(g_{B_{1}}+g_{D_{0}}+g_{A_{0}}\right) \hat{X}, \\
& \langle 5|H| 5\rangle=\epsilon_{D_{0}}+\epsilon_{A_{1}}+\epsilon_{B_{0}}+\hat{Y}+\left(g_{D_{0}}+g_{A_{1}}+g_{B_{0}}\right) \hat{X}, \\
& \langle 6|H| 6\rangle=\epsilon_{A_{1}}+\epsilon_{B_{1}}+\epsilon_{D_{1}}+\hat{Y}+\left(g_{B_{1}}+g_{A_{1}}+g_{D_{1}}\right) \hat{X}, \\
& \langle 7|H| 7\rangle=\epsilon_{D_{0}}+\epsilon_{B_{1}}+\epsilon_{A_{1}}+\hat{Y}+\left(g_{B_{1}}+g_{D_{0}}+g_{A_{1}}\right) \hat{X}, \\
& \langle 8|H| 8\rangle=\epsilon_{D_{1}}+\epsilon_{B_{0}}+\epsilon_{A_{1}}+\hat{Y}+\left(g_{B_{0}}+g_{D_{1}}+g_{A_{1}}\right) \hat{X}, \\
& \langle 4|H| 5\rangle=\langle 5|H| 4\rangle=J_{A B}, \\
& \langle 6|H| 8\rangle=\langle 8|H| 6\rangle=J_{A B}, \\
& \langle 5|H| 4\rangle=\langle 4|H| 5\rangle=J_{B D}, \\
& \langle 7|H| 8\rangle=\langle 8|H| 7\rangle=J_{B D}, \\
& \langle 3|H| 5\rangle=\langle 5|H| 3\rangle=J_{A D}, \\
& \langle 6|H| 7\rangle=\langle 7|H| 6\rangle=J_{A D} .
\end{aligned}
$$

For $\epsilon_{A 0}=\epsilon_{B 0}=\epsilon_{D 0}=\epsilon$ and $J_{A B}=J_{B D}=J_{A D}=J$ the eigenvalues are given by

$$
\begin{aligned}
& E_{1}=0, \\
& E_{2}=3 \epsilon, \\
& E_{3}=\epsilon-J, \\
& E_{4}=\epsilon+2 J, \\
& E_{5}=2 \epsilon-J, \\
& E_{6}=2 \epsilon+2 J .
\end{aligned}
$$

For the chain as in Fig. 3] we set $J_{A D}=0$ and $J_{A B}=$ $J_{B D}=J$. The resulting eigenvalues are:

$$
E_{1}=0
$$




$$
\begin{aligned}
& E_{2}=3 \epsilon, \\
& E_{3}=2 \epsilon, \\
& E_{4}=\epsilon, \\
& E_{5}=\epsilon \pm \sqrt{2} J \\
& E_{6}=2 \epsilon \pm \sqrt{2} J .
\end{aligned}
$$

In the subspace with only one exciton, the basis consists of the three states $|1,0,0\rangle,|0,1,0\rangle$, and $|0,0,1\rangle$, and the matrix reduces to

$$
M=\left(\begin{array}{ccc}
\epsilon_{D}+\hat{Y}+2 \hat{X} & J_{A B} & J_{A D} \\
J_{A B} & \epsilon_{B}+\hat{Y} & J_{B D} \\
J_{A D} & J_{A B} & \epsilon_{A}+\hat{Y}-2 \hat{X}
\end{array}\right) .
$$

The eigenvalues for the trimer as in Fig. 2 are

$$
\begin{aligned}
& E_{1}=\epsilon-J, \\
& E_{2}=\epsilon+2 J,
\end{aligned}
$$

and for the chain as in Fig. 3

$$
\begin{aligned}
& E_{1}=\epsilon, \\
& E_{2}=\epsilon \pm \sqrt{2} J
\end{aligned}
$$

The model is equivalent to eq. (22) with $N=3$.

\section{Multi-site exciton-boson model}

In the single-exciton subspace, the fermionic degrees of freedom of the models introduced above can be mapped onto operators $a_{i}^{(\dagger)}$ for hard-core bosons corresponding to the creation and annihilation of an exciton at site $i$. This results in general multi-site exciton-boson models with $N$ sites defined by

$$
H_{\text {multi }}=H_{\mathrm{x}}+H_{\mathrm{x}-\text { bath }}+H_{\mathrm{bath}}
$$

with the electronic part defined as

$$
H_{\mathrm{x}}=\sum_{i, j}^{N} J_{i j} a_{i}^{\dagger} a_{j}
$$

The parameters $J_{i j}$ for $i \neq j$ are the transfer integrals between site $i$ and $j$. As before, we only consider nearest neighbour interactions. The diagonal elements $J_{i i}$ are the on-site energies $\varepsilon_{i}$ at site $i$. We perform a constant shift of the Hamiltonian by $J_{i i}=J_{j j}(\forall i, j)$ and arrive at

$$
H_{\mathrm{x}}=\sum_{i, j, i \neq j}^{N} J_{i j} a_{i}^{\dagger} a_{j}
$$

For the coupling term we assume the following form

$$
H_{\mathrm{x}-\mathrm{bath}}=\sum_{i}^{N} g_{i} a_{i}^{\dagger} a_{i} \sum_{n} \lambda_{n}\left(b_{n}^{\dagger}+b_{n}\right)
$$

with $g_{i}=(i-(N+1) / 2)$. For $N=2$ the model is equivalent to the spin-boson model with the matrix $M$ as in eq. (15) and for $N=3$ equivalent to eq. (19).

The eigenvalues of $H_{\mathrm{x}}$ for $N=4$ are $E=0, \pm 2 J$ for the ring geometry and $E= \pm J$ for the chain. The eigenvalues for the ring with $N=5$ are $E=2 J,(\sqrt{5}-$ 1) $J / 2,(-\sqrt{5}-1) J / 2$ and for the ring with $N=6$ : $E= \pm J, \pm 2 J$.

\section{METHOD}

The models we are considering here are completely specified by the parameters of the electronic system and the spectral function $J(\omega)$ (defined in eq. (7)) which can be estimated in a classical molecular dynamics simulation. We are using here an ohmic form:

$$
J(\omega)=2 \pi \alpha \omega \Theta\left(\omega-\omega_{c}\right)
$$

where $\alpha$ is the dimensionless coupling for which we use values in the range $0.01-2$. The parameters $\mathrm{J}$ and $\mathrm{t}$ are all measured in units of $\omega_{c}$. Typical values of $\hbar \omega_{c}$ are of the order of 1 to $10 \mathrm{meV}$.

As described in the introduction, basically all degrees of freedom of the bosonic bath (the dissipative environment) are relevant for the behaviour of the electronic or excitonic system. So it is not possible to disregard high energy states even if we are interested in low temperature properties like the coherent behaviour for temperatures smaller than the characteristic temperature $T^{*}$. The renormalization group ansatz is designed for problems where every energy scale contributes and perturbation theory typically shows logarithmic divergencies at small frequencies (energies) when the temperature goes to zero.

In order to keep the paper self contained we explain the Numerical Renormalization Group (NRG) method for the bosonic bath in detail.

Originally the NRG was invented by Wilson for a fermionic bath to solve the Kondo problem [25, 26]. The fermionic NRG is a standard and powerful tool to investigate complex impurity problems with one or more fermionic baths. Only recently, the method was extended to treat quantum impurity systems with a coupling to a bosonic bath [27, 28]. Here we focus on equilibrium quantities (recently it was shown that the NRG can also be applied to non-equilibrium situations 29].)

For the numerical renormalization procedure we start from the Hamiltonian written in a continous form (see the detailed discussion in Ref. 28):

$$
H=H_{\mathrm{el}}+\int_{0}^{1} d \varepsilon g(\varepsilon) b_{\varepsilon}^{\dagger} b_{\varepsilon}+\left(\sum_{i} g_{i} n_{i}\right) \int_{0}^{1} d \varepsilon\left(b_{\varepsilon}^{\dagger}+b_{\varepsilon}\right)
$$




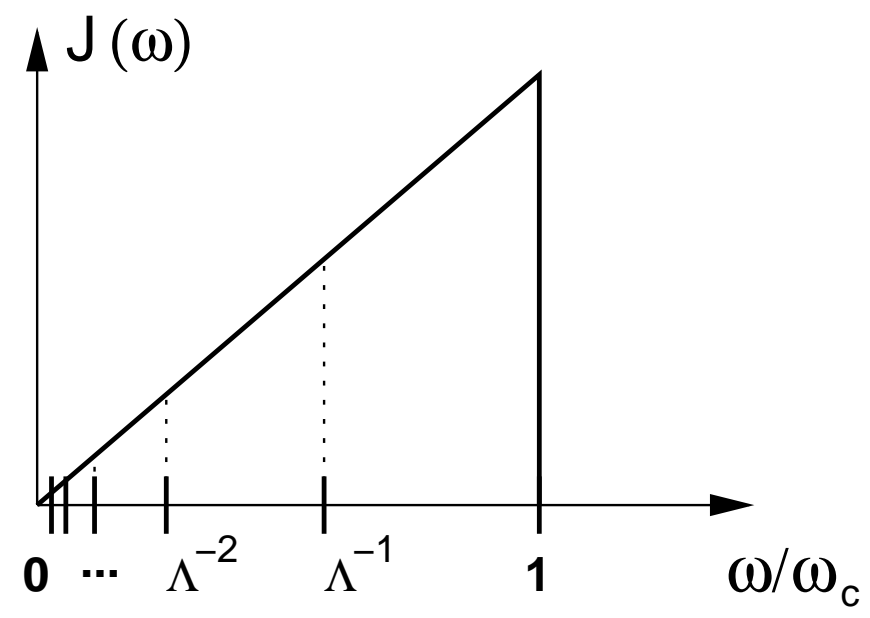

FIG. 4: Logarithmic discretization of the spectral function $J(\omega)$.

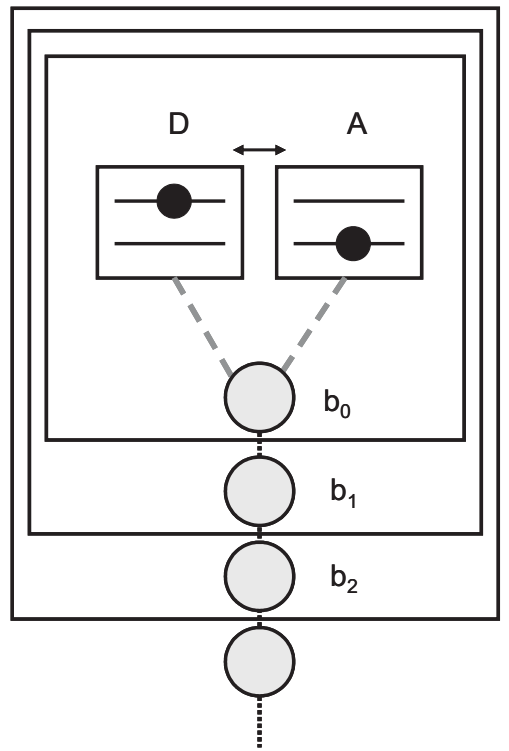

FIG. 5: Scheme of the bosonic chain-NRG. The boxes represent the iterative diagonalization.

The function $g(\varepsilon)$ is the dispersion of the phonon bath and $h(\varepsilon)$ characterizes the coupling between the electronic system and the bath. Both functions are related to the spectral function $J(\omega)$, see Ref. 28. We start defining the renormalization group transformations by a logarithmic discretization of the bath spectral function (Fig. (4) in intervals $\left[\Lambda^{-n+1}, \Lambda^{-n}\right]$, with $n=0,1, \ldots, \infty$ and $\Lambda>1$ the NRG discretization parameter. The discretization is exact for $\Lambda \rightarrow 1$ and works still very well for $\Lambda=3$. (Here we are using $\Lambda=2$ )

Within each of these intervals only one bosonic degree of freedom is retained as a representative of the continuous set of degrees of freedom. The function $h(\varepsilon)$ is cho- sen to be a constant in each intervall of the logarithmic discretization. The Hamiltonian is written in the new discrete basis and the resulting Hamiltonian is mapped onto a semi-infinite chain (Fig. (5) with the electronic part $H_{\text {el }}$ coupling to the first site of the bosonic chain. Finally the chain-Hamiltonian is numerically diagonalized via successively adding one site to the chain. The effective Hamiltonian is treated on successive smaller energy scales by the renormalization group transformation

$$
\begin{aligned}
H_{N+1} & =\Lambda H_{N} \\
& +\Lambda^{N+1}\left[\varepsilon_{N+1} b_{N+1}^{\dagger} b_{N+1}+t_{N}\left(b_{N}^{\dagger} b_{N+1}+\text { h.c. }\right)\right] .
\end{aligned}
$$

The energies $\varepsilon_{n}$ and couplings between the elements of the chain are falling off as $\Lambda^{-n}$.

The bosonic NRG has been shown to give very accurate results for the spin-boson model [27, 28]. One of its strengths is the flexibility to handle a variety of models involving the coupling of a small subsystem to a bosonic bath.

To detect possible phase transitions, we calculate the eigenvalue spectrum and the density-density correlation function (see below). In the limit of $\alpha=0$ the exciton system and the bosonic degrees of freedom are completely decoupled. The coherent motion of the exciton is undamped and we are in the delocalized phase. In contrast, in the case of $J=0$ the system is in the localized phase. The two phases (localized and delocalized) are connected by a quantum phase transition. Similar to the analysis in Ref. 28, the phase diagrams of the exciton-boson models studied here can be obtained from the flow diagram of the lowest-lying many-particle levels. Another possibility is to calculate the density-density autocorrelation function $C(\omega)$ which shows a divergency at the phase transition.

We calculate $C(\omega)$ for the dimer and trimer for different sets of parameters. This quantity is defined by

$C(\omega)=\frac{1}{4 \pi} \int_{-\infty}^{\infty} d t e^{i \omega t}\left\langle\left[\sum_{i} g_{i} a_{i}^{\dagger} a_{i}(t), \sum_{i} g_{i} a_{i}^{\dagger} a_{i}(0)\right]\right\rangle$,

and probes the dynamics under equilibrium preparation. For the two-site model, $C(\omega)$ corresponds to the spin-spin correlation function of the equivalent spin-boson model

$$
C(\omega)=\frac{1}{4 \pi} \int_{-\infty}^{\infty} d t e^{i \omega t}\left\langle\left[\sigma_{z}(t), \sigma_{z}(0)\right]\right\rangle
$$

with $\sigma_{z}$ the $z$-component of the spin in this model.

We calculate the density-density correlation function as sum of $\delta$-functions in the Lehmann representation:

$$
C(\omega)=\frac{1}{2} \sum_{n}\left|\left\langle 0\left|\sum_{i} g_{i} a_{i}^{\dagger} a_{i}\right| n\right\rangle\right|^{2} \delta\left(\omega+\epsilon_{0}-\epsilon_{n}\right),
$$$$
\omega>0 \text {. }
$$ 
The linear absorption and emission coefficient $\alpha(\omega)$ for the donor site of the electronic system coupled to the bosonic bath under influence of an external laser field of frequency $\omega$ is given by Fermi's golden rule:

$$
\alpha^{D}(\omega)=2 \pi \sum_{f}\left|\left\langle f\left|H_{\text {pert }}^{D}\right| 0\right\rangle\right|^{2} \delta\left(\omega+E_{0}-E_{f}\right),
$$

where $H_{\text {pert }}^{D}$ is defined as

$$
H_{\text {pert }}^{D}=c_{D 1}^{\dagger} c_{D 0}+c_{D 0}^{\dagger} c_{D 1} .
$$

The term $H_{\text {pert }}^{D}$ describes the excitation of an electron from the ground state $D_{0}$ to the excited state $D_{1}$. It can be treated perturbatively as long as the probing photon energy is small. For the initial state we use the ground state $|0\rangle$ and $|f\rangle$ are all possible final states.

The eigenenergies of $H\left(E_{0}\right.$ and $\left.E_{f}\right)$ and the matrix elements $\left\langle 0\left|H_{\text {pert }}\right| f\right\rangle$ are evaluated with the NRG for different $J$ and increasing coupling to the bosonic bath. To obtain a continuous curve for $\alpha^{D}(\omega)$, the $\delta$-functions appearing in eq. 28 have to be broadened. Here we use the strategy discussed in Ref. 30, that is replacing each $\delta$-function by a gaussian on a logarithmic scale. On a linear scale, this function is not symmetric around its center so that spectral weight in $\alpha^{D}(\omega)$ appears to be shifted to higher frequencies.

\section{RESULTS}

\section{Phase Diagram}

Increasing $J$ tends to delocalize the exciton. Since we have excluded the single-electron hopping in the Hamiltonian, the dynamics can be restricted to the single-exciton subspace which maps onto a spin-boson model for two sites. For increasing coupling to the bosonic bath, the exciton localizes at a critical $\alpha_{\mathrm{c}}$. We explore the phase diagram with localized and delocalized phases (connected by a Kosterlitz-Thouless transition) for the exciton-boson model with 2 to 6 molecular sites by calculating the critical $\alpha_{c}(J)$ as a function of $J$. Note that for a finite bias $\varepsilon$ (difference of the excitation energy on donor and acceptor) there is no phase transition but a crossover from a delocalized to a more localized regime.

The phase diagram for the spin-boson model was calculated already in Ref. 28. The critical $\alpha$ depends linearly on the matrix element $J$. It was noted that the exact value of $\alpha_{c}$ has to be determined in the limit of $\Lambda \rightarrow 1$. We do not perform the extrapolation so that the critical $\alpha_{c}$ is somewhat larger than the actual value.

In Fig. 6] we display the phase diagram of the multi-site exciton-boson model for $3,4,5$ and 6 sites for both chain and ring geometry.

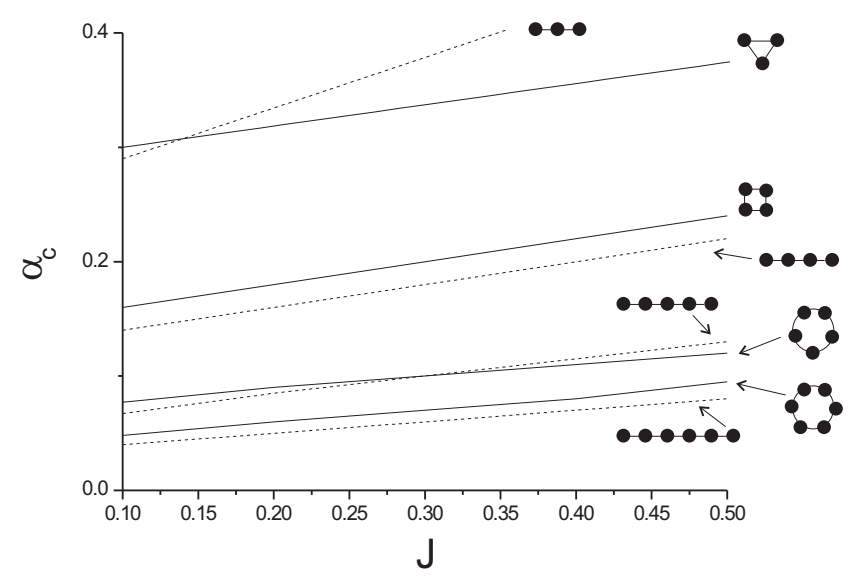

FIG. 6: Phase diagram of rings (solid line) and chains (dotted line) with $3,4,5$, and 6 sites. The system is in the localized (delocalized) phase above (below) the phase boundary.

The dashed and solid lines display the critical $\alpha_{c}$ for the chain and ring, respectively. The critical coupling shows a linear behaviour similar as in the spin-boson model. For an even number of sites the ring has a larger critical $\alpha$ than the chain. For an odd number of sites, both curves cross at a certain value of $J$ above which the opening of the ring will tends to delocalize the exciton.

For a multi-site exciton-boson model with three or more sites, no quantum phase transition is observed as soon as the couplings $J_{i j}$ between neighbouring sites are different. To study the crossover from the delocalized to a more localized phase, we calculate equilibrium dynamical properties as discussed in the following.

\section{Equilibrium Dynamical Properties}

To study the dynamics of the electron transfer process in the one-exciton subspace we calculate the densitydensity correlation function $C(\omega)=\frac{1}{2 \pi} \int_{-\infty}^{+\infty} e^{i \omega t} C(t) \mathrm{d} t$ with

$$
C(t) \sim\left\langle\left[a_{D}^{\dagger} a_{D}(t)-a_{A}^{\dagger} a_{A}(t), a_{D}^{\dagger} a_{D}(0)-a_{A}^{\dagger} a_{A}(0)\right]_{+}\right\rangle,
$$

for the dimer $\left(g_{D}=1 / 2, g_{A}=-1 / 2\right)$ and for the trimer $\left(g_{B}=0, g_{A}=-1, g_{D}=1\right)$.

In the two-site case, the density-density correlation function is identical to the spin-spin correlation function. The correlation function shows the power-law behaviour for low frequencies up to $\omega \approx T^{*}$. When $\alpha$ approaches $\alpha_{\mathrm{c}}$, the slope in $C(\omega)$ increases and the peak position (the characteristic energy and temperature scale $T^{*}$ ) is shifted to lower energies, see Fig. [7 At the phase transition the correlation function is diverging. The correlation func- 


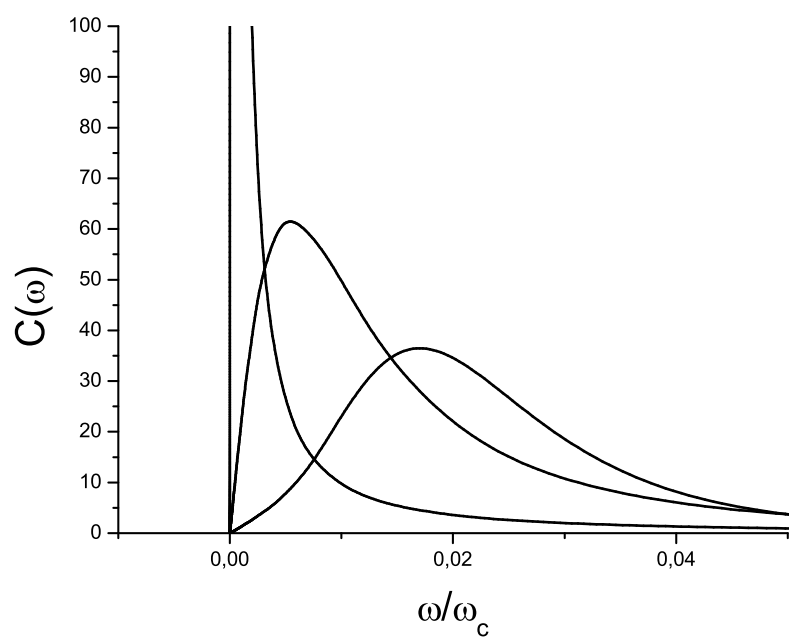

FIG. 7: Density-density correlation function $C(\omega)$ for the dimer for different $\alpha$ and $J=0.4$. For small frequencies, $C(\omega)$ is linear in $\omega$. The slope increases with increasing $\alpha=0.1,0.2,0.3$. For zero bias $(\varepsilon=0)$ the phase transition to the localized phase is indicated by an infinite slope.

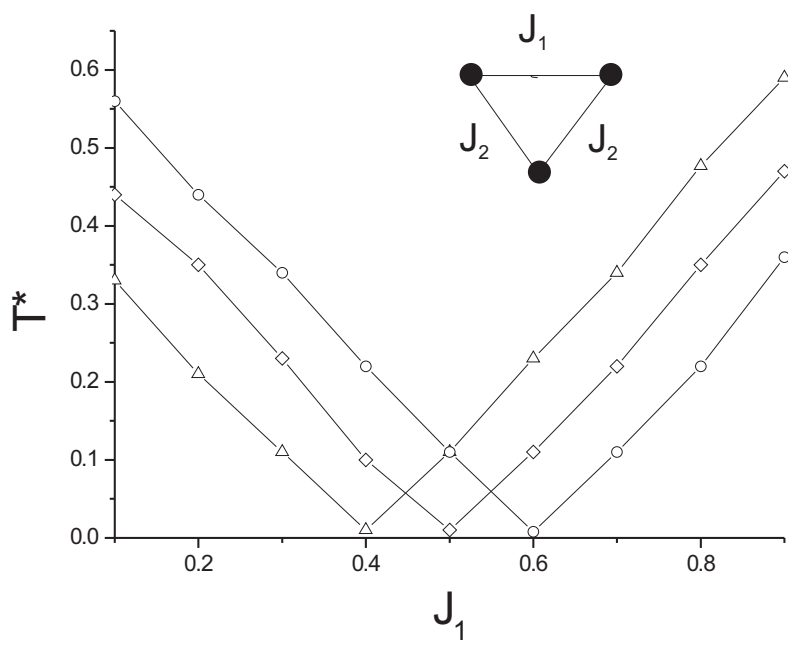

FIG. 8: Crossover temperature for a trimer ring with $J_{2}=$ $J_{A B}=J_{D B}=0.4$ (tiangles), 0.5 (diamonds), 0.6 (circles) and various values of $J_{1}$. The coupling $\alpha$ is set to 0.1

tion shows an algebraic long time behaviour for $T=0$ and an exponential decay for finite $T$.

In Fig. 8 we show results for the characeristic temperature for a trimer ring by keeping $J_{A B}$ and $J_{D B}$ constant and varying $J_{1}=J_{D A}$. $\left(J_{D A}=0\right.$ corresponds to the chain.). The value of $T^{*}$ goes through a minimum at $J_{1}=J_{A B}=J_{D B}$. The larger the difference the larger is the characteristic temperature. In Fig. 9 we display

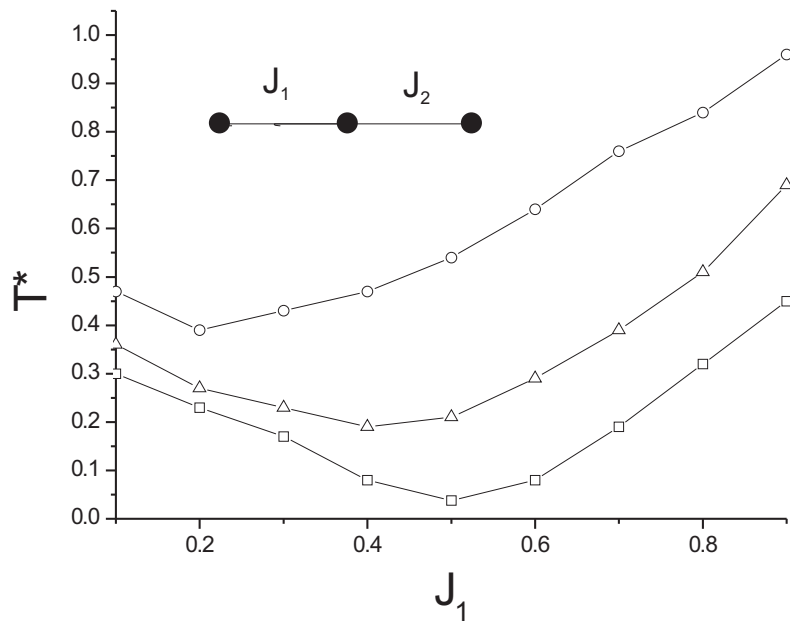

FIG. 9: Crossover temperature for a trimer chain with $J_{2}=$ 0.5 and various values of $J_{1}$. The coupling $\alpha$ is set to $0.1,0.2$ and 0.3 for the upper, middle and lower curve, respectively

the characteristic temperature for an asymmetric threesite chain with $J_{1} \neq J_{2}$ for increasing $\alpha$. For large $\alpha$ and $J_{1}=J_{2}$ the characteristic temperature $T^{*}$ goes to zero indicating the phase transition. For the asymmetric chain no phase transition occurs and $T^{*}$ increases with the increasing difference of the matrix elements $J_{1}$ and $J_{2}$.

\section{Absorption Spectrum}

For $\alpha=0$ the spectrum of the two-site electron-boson model consists of four states. In the ground state, both electrons occupy the lowest level of donor and acceptor molecules, respectively. The system can be excited by a photon: $D+A \rightsquigarrow D^{*}+A$. If we now consider a finite $J$, the exciton is able to move to the acceptor and back $\left(D^{*}+A \leftrightarrows D+A^{*}\right)$

To calculate the absorption spectrum we choose the intitial state to be the ground state. The ground state is calculated with the NRG and depends on $\alpha$ and $J$. We set the energy difference between the ground state and the excited state to $\varepsilon=0.75 \omega_{c}$. For $\alpha=0$ and $J=0$ the peak in the absorption spectrum is at $\omega=\varepsilon=$ 0.75 . For increasing $J$ peaks are at frequencies equal to the eigenenergies $\omega=\varepsilon \pm J$. If now $\alpha$ is increased the two main peaks are broadened and shifted (see Fig. 10). The height of the peak at low frequencies increases with increasing $\alpha$.

The absorption spectrum for the trimer with $\alpha=0$ shows peaks at $\varepsilon+2 J$ and $\varepsilon-J$ for the ring geometry (with $J_{A B}=J_{A D}=J_{B D}=J$ ) and at $\varepsilon \pm \sqrt{2} J$ for the chain. The absorption spectra for various values of 


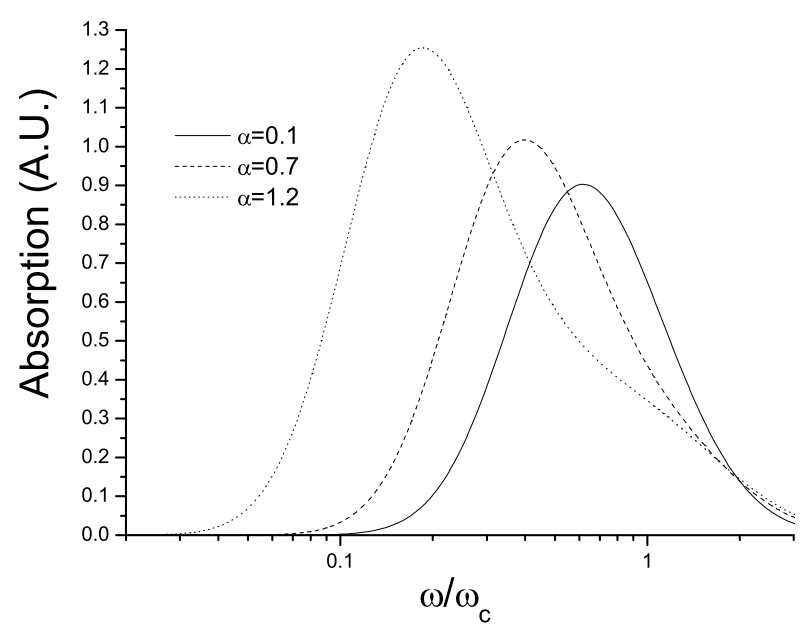

FIG. 10: Dimer absorption spectrum for $J=0.2, \epsilon_{D}=\epsilon_{A}=$ $0.75 \omega_{c}$ and $\alpha=0.1,0.7,1.2$ as a function of $\omega$.

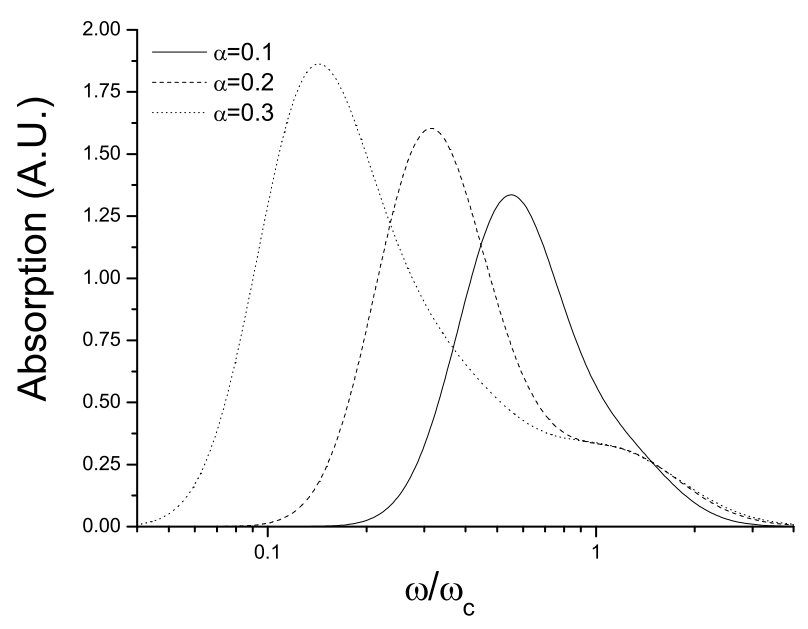

FIG. 11: Absorption spectrum of a trimer ring for $J=0.2$, $\epsilon_{D}=\epsilon_{A}=0.75 \omega_{c}$ and $\alpha=0.1,0.2,0.3$ as a function of $\omega$.

$\alpha$ are shown in Figs. 11 and 12 for the ring and chain, respectively.

\section{CONCLUSION}

In this paper we studied the phase diagram, equilibrium dynamical properties and the linear absorption spectrum of Frenkel excitons in various ring and chain models with a coupling of the electronic degrees of freedom to a bosonic bath. We used the numerical renormalization group method which allows to study the electronboson and exciton-boson models in the full parameter regime.

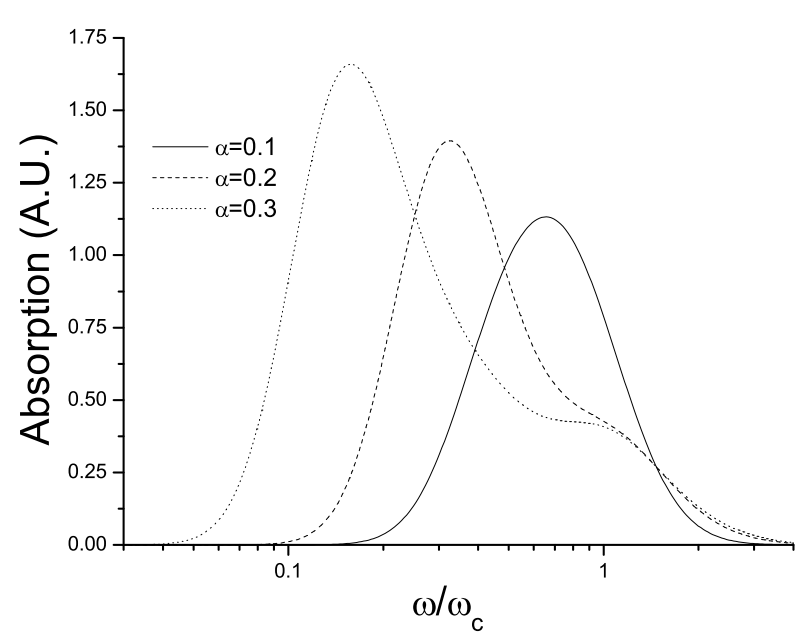

FIG. 12: Chain absorption spectrum for $J=0.3, \epsilon_{D}=\epsilon_{A}=$ $0.75 \omega_{c}$ and $\alpha=0.1,0.2,0.3$.

We studied in detail the phase diagrams of the multisite electron-boson models in the subspace of one exciton. In the zero-bias case (all molecules have degenerate HOMO and LUMO energies), increasing the value of $\alpha$ leads to a quantum phase transition between a delocalized and a localized phase. For the two-site case (dimer) the exciton-boson model can be mapped onto the spin-boson model for which the phase diagram is already known. For more than two sites, the behaviour is more complicated and depends also on the geometry (chain vs. ring).

The calculation of the density-density correlation function allows to estimate the characteristic temperature for the crossover between delocalized and localized phase. This crossover temperature $T^{*}$ is zero for the localized phase and increases when the system goes to the delocalized phase. Increasing the difference between the various couplings of the model generally leads to a more delocalized behaviour.

It would be interesting to compare our results to optical experiments of small bio-engineered systems or quantum dots in which exciton transfer occurs. Further studies are planned to evaluate the time dependent behaviour of excitons, to extend the system to larger rings and to include static disorder using time dependent and equilibrium NRG to model systems like the LH II ring.

\section{Acknowledgement}

We acknowledge helpful discussions with U. Kleinekathöfer, M. Wubs, and D. Vollhardt. This research was supported by the DFG through SFB 484 (ST, RB), the Center for Functional Nanostructures 
(NT), and by the Alexander von Humboldt foundation $(\mathrm{NT})$.

[1] Hu X, Ritz T, Damjanovic A, Autenrieth F, and Schulten K 2002 Quarterly Reviews of Biophysics 35, 1-62

[2] Agranovich V M and Bassani G F 2003 Electronic Excitations in Organic Based Nanostructures, Thin Films and Nanostructures 31 (Amsterdam: Elsvier)

[3] May V and Kühn O 2004 Charge and Energy Transfer Dynamics in Molecular Systems (Weinheim: Wiley)

[4] Fleming G R and Scholes G D 2004 Nature 431256

[5] Nazir A, Lovett B W, and Briggs G A D 2004 optical Stark effect Phys. Rev. A $\mathbf{7 0} 052301$

[6] Danjanovic A, Ritz T, and Schulten K 1999 Phys. Rev. E 593293

[7] Davydov A S 1962 Theory of Molecular Excitons (New York: McGraw-Hill)

[8] Jordanides X J, Scholes G D, Shapley W A, Reimers J R and Fleming G R 2004 J. Phys. Chem. B 1081753

[9] Mukamel S Principles of Nonlinear Optical Spectroscopy 1995 (New York: Oxford)

[10] Leggett A J, Chakravarty S, Dorsey A T, Fisher M P A, Garg A, and Zwerger W 1987 Rev. Mod. Phys. 591

[11] Weiss U 1999 Quantum dissipative systems, 2nd ed. (Singapore: World Scientific)

[12] Haken H and Reineker P 1972 Z. Phys. 249, 253; Haken $\mathrm{H}$ and Strobl S 1973 Z. Phys. 262135

[13] Agranovich V M and Galanin M D 1982 Electronic Excitation Energy Transfer in Condensed Matter (Amsterdam: Elsvier)
[14] Cheng Y C and Silbey R J 2006 Phys. Rev. Lett. 96 028103

[15] Wubs M and Knoester J 1998 Chem. Phys. Lett. 28463

[16] Herman P and Barvik I 2001 Chem. Physics 274199

[17] Kleinekathöfer U, Schröder M and Schreiber M 2005 Journal of Lumin 112461

[18] Damjanovich A, Kosztin I, Kleinekathöfer U and Schulten K 2002 Phys. Rev. E 65, 031919

[19] Redfield A G 1965 Adv. Magn. Reson. 11

[20] Di Felice R A. Calzolari A, Varsano D, Rubio Secades A 2006 in Introducing Molecular Electronics edited by Cuniberti G Richter K and Fargas G (Heidelberg: Springer)

[21] Starikov E B 2003 Phil. Mag. Lett. 83699

[22] Gilmore J and McKenzie R H 2005 J. Phys.: Condens. Matter 171735

[23] Xu D and Schulten K 1994 Chem. Phys. 182, 91

[24] Tornow S, Tong N H and Bulla R 2006 Eur. Phys. Lett. to appear

[25] Wilson K G 1975 Rev. Mod. Phys. 47773

[26] Krishna-murthy H R, Wilkins J W, and Wilson K G 1980 Phys. Rev. B 21 1003; ibid. 211044

[27] Bulla R, Tong N H, and Vojta M 2003 Phys. Rev. Lett. 91, 170601

[28] Bulla R, Lee H J, Tong N H and Vojta M 2005 Phys. Rev. B $\mathbf{7 1} 045122$

[29] Anders F B and Schiller A 2005 Phys. Rev. Lett. 95 196801

[30] Bulla R, Costi T A, and Vollhardt A 2001 Phys. Rev. B 64045103 